\title{
O papel e a importância do médico veterinário no sistema único de saúde: uma análise à luz do direito sanitário
}

The veterinarian role and importance into the unified health system: an analysis under the sanitary law

El papel y la importancia del veterinario en el sistema único de salud: una revisión bajo el derecho sanitario

Nino Tollstadius Armelin 1 Jarbas Ricardo Almeida Cunha

RESUMO. Introdução: o campo da Medicina Veterinária é vasto e as oportunidades para a saúde pública veterinária são ilimitadas. Entretanto, o seu maior desafio é ser capaz de aplicar a grande quantidade de resultados de pesquisas disponíveis e o conhecimento técnico dotado para melhorar a desigualdade social, contribuindo para o desenvolvimento nacional. $\mathrm{O}$ aumento da incidência das doenças infecciosas e parasitárias fez com que o médico veterinário se tornasse peça fundamental no controle dessas doenças e na manipulação de fatores ambientais que interagem com a vida das pessoas. Este trabalho tem o objetivo de apresentar o papel e a importância do médico veterinário no Sistema Único de Saúde, com base na legislação em vigor. Metodologia: tratou-se de um estudo de natureza qualitativa utilizando a análise bibliográfica e legislativa. Resultados e conclusão:o médico veterinário possui um conhecimento interdisciplinar e é importante no contexto da saúde pública por atuar na prevenção e promoção da saúde humana. no SUS, o médico veterinário pode atuar nas vigilâncias em saúde, nas comissões intersetoriais e permanente e nas direções nacional, estadual e municipal, presentes nos artigos $6^{\circ}$ e 12 ao 18 da Lei Orgânica da Saúde.

Palavras-chave: Saúde Pública, Médico Veterinário, Vigilância em Saúde

ABSTRACT. Introduction: The Veterinarian Medicine field is extensive and the opportunities to the veterinarian health public are limitless. However, its biggest challenge is being capable of apply the large amount of research results available and the technical knowledge acquired to improve de social inequity, contributing to the national development. The increasing incidence of infectious, parasitic disease meant that the veterinarian became a fundamental piece on these diseases control and the environmental factors manipulation that interact with the people's life. This paper aims to present the veterinarian role and importance into the Unified Health System, considering the legislation in force. Methodology: it was a qualitative study that used the bibliographic and legislative analysis. the veterinarian has an interdisciplinary knowledge being important on the public health context for working in the prevention and promotion

\footnotetext{
${ }^{1}$ Graduado em Medicina Veterinária pela UPIS. E-mail: nino.armelin@gmail.com.

${ }^{2}$ Graduado em Direito pela Universidade Federal de Ouro Preto - UFOP. Especialização em Direito Sanitário pela Fundação Oswaldo Cruz - Fiocruz Brasília. Mestrado em Política Social pela Universidade de Brasília - UnB. Analista Técnico de Políticas Sociais (ATPS) no Ministério do Desenvolvimento Social e Combate à Fome (MDS). Professor de Direito Constitucional Sanitário. E-mail: jarbas.ricardo@yahoo.com.br.
} 
of the human health. Results and conclusion: on SUS, the veterinarian can work on the health surveillance, on the inter sector and permanent commissions, and on the national, state, municipal directions, present on the articles $6^{\circ}$ and 12 to 18 of the Health Organic Law.

Keywords: Public Health, Veterinarian, Health Surveillance

RESUMEN. Introducción: el campo de la medicina veterinaria es enorme y las oportunidades para la salud pública veterinaria son ilimitadas. Sin embargo, su mayor reto es ser capaz de aplicar una gran cantidad de resultados de investigaciones disponibles y la experiencia prevista para mejorar la desigualdad social, contribuyendo al desarrollo nacional. El aumento de la incidencia de enfermedades infecciosas y parasitarias hizo que el veterinario se convirtiera en un elemento fundamental en el control de esas enfermedades y en la manipulación de los factores ambientales que están vinculados con la vida de las personas. Este trabajo tiene como objetivo presentar el papel y la importancia del veterinario en el Sistema Único de Salud, con base en la legislación vigente. Metodología: este fue un estudio cualitativo utilizando el análisis bibliográfico y legislativo. El veterinario tiene un conocimiento interdisciplinar y es importante en el contexto de la salud pública por actuar en la prevención y en la promoción de la salud humana, a través de la presentación de informes y del control de las zoonosis, de la inspección y de la fiscalización sanitaria de productos de origen animal y de programas de educación sanitaria y del medio ambiente. Resultados e conclusiones: en el SUS, el veterinario puede actuar en la prevención de enfermedades, en las Comisiones Intersectoriales y Permanentes, y en las Direcciones Nacional, Estatal y Municipal presentes en los artículos 6 y 12 al 18 de la Ley Orgánica de Salud.

Palabras-llave: Salud pública, Veterinario, Vigilancia de la Salud

\section{Introdução}

A Medicina Veterinária no Brasil surgiu por volta de 1810, pelo Conde de Linhares, Ministro de Estado dos Negócios Estrangeiros da Guerra, que criou o primeiro cargo de Veterinário para orientar tecnicamente $01^{\circ}$ Regimento de Cavalaria do Exército. $O$ ensino de Veterinária no Brasil não possui uma data exata, devido à divergência entre as datas de fundação das escolas veterinárias e seu início de funcionamento. A prioridade é a Escola de Agronomia e Veterinária de Pelotas/RS, com a inauguração em 08 de dezembro de 1883 (1).

A princípio, a Medicina Veterinária surgiu como uma área de conhecimento para promover a saúde dos animais com o intuito de diminuir os prejuízos econômicos gerados pelas patologias que os acometiam (2). Com o passar do tempo, percebeu-se que muitas doenças infecciosas humanas eram transmitidas a partir de reservatórios 
animais e de alimentos contaminados, o que levou a prática veterinária a se voltar para aos aspectos populacionais e preventivos da saúde pública, por meio dos conhecimentos epidemiológicos e melhoria na produção de alimentos (3).

O médico veterinário é um profissional da saúde a serviço do homem, tanto por suas ações de prevenção e controle das zoonoses como por seus serviços de promoção na produção de alimentos, buscando sempre a melhor qualidade de seus produtos para o consumo humano. Além disso, sintetiza e enumera as responsabilidades do veterinário na comunidade: a) higiene dos alimentos; b) saneamento ambiental; c) promoção da saúde animal; e d) controle de zoonoses (4).

O Sistema Único de Saúde (SUS) brasileiro, atualmente, apresenta diversos modelos assistenciais, entre eles o modelo médico-assistencial privatista com destaque na assistência médico-hospitalar, nos serviços de apoio diagnóstico e terapêutico e o modelo sanitarista que se promove por meio de campanhas, programas especiais e ações da Vigilância Epidemiológica e Sanitária (5).

O modelo médico-assistencial tem como sujeito o médico e a doença como objeto, em sua expressão individualizada, tomando como meios de trabalho os conhecimentos e tecnologias que permitem o diagnóstico e a terapêutica das diversas patologias. Em contrapartida, o modelo sanitarista possui como sujeito o sanitarista, qualquer profissional da saúde cujo trabalho adota por objeto os modos de transmissão e fatores de risco das diversas doenças, por meio da perspectiva epidemiológica, utilizando um conjunto de meios que compõem a tecnologia sanitária (educação em saúde, saneamento, controle de vetores, imunização, entre outras) (5).

O SUS, regulamentado pela Lei $8.080 / 1990^{3}$, define no artigo $2^{\circ}$ que a saúde é um direito do ser humano, conforme estabelece a Carta Magna, e o Estado tem como dever prover as condições indispensáveis ao seu pleno exercício. No artigo 6º , estão previstas as ações de vigilância sanitária e epidemiológica; de saúde do trabalhador; fiscalização e a inspeção de alimentos, água e bebidas para consumo humano (6).

\footnotetext{
${ }^{3} \mathrm{O}$ Brasil, devido os vetos de Collor do texto original da Lei 8.080/90, origina talvez o único exemplar conhecido de uma Lei Orgânica da Saúde, oriunda de uma política social que, na verdade, são duas leis: a lei 8.080/90, que não foi aprovada em toda sua integralidade e a lei 8.142/90, aprovada três meses depois, com a marca da resistência e mobilização em torno das bandeiras iniciais da Reforma Sanitária para ratificar os propósitos da lei 8.080, como a criação dos conselhos para a fiscalização dos recursos repassados pelos entes federados (9).
} 
A reforma sanitária brasileira (RSB) surgiu na década de 1970 e foi um dos processos mais importantes na história das políticas de saúde no Brasil. A RSB desembocou na $8^{\text {a }}$ Conferência Nacional de Saúde (8 ${ }^{\underline{a}}$ CNS), sendo formalizada na Constituição da República de 1988, e consolidou-se através de lutas sociais que buscavam a melhoria das condições de vida e de saúde para todos os brasileiros (7).

A Constituição Federal brasileira de 1988 introduziu a saúde no capítulo II que faz referência à seguridade social. Os artigos 196 a 200 trouxeram grandes inovações para a saúde pública brasileira, como a universalidade do acesso, a integralidade e a equidade da atenção, a descentralização das ações e aplicações de recursos mínimos aos serviços públicos de saúde. Outro marco importante foi a ampliação decisiva da participação da sociedade na discussão, na formulação e no controle das políticas públicas de saúde (8).

A profissão de médico veterinário durante muitos anos ficou restrita à agricultura, e como consequência muitas zoonoses ${ }^{4}$ ainda são controladas ou erradicadas sob a perspectiva econômica, da mercadoria animal, e não do ponto de vista da saúde pública (10).

Identificando a necessidade de conciliar os fundamentos da saúde humana com a saúde animal, a Organização Mundial da Saúde (OMS), em 1946, propôs a criação de uma seção da saúde veterinária. Durante a conferência da OMS, em 1951, foi utilizada pela primeira vez a expressão Saúde Pública Veterinária, compreendendo que "todos os esforços da comunidade que influenciam e são influenciados pela arte e ciência médico veterinária, aplicados à prevenção da doença, proteção da vida e promoção do bemestar e eficiência do ser humano" marcam o conceito da participação do veterinário na saúde pública e o qualifica para algumas atribuições, sendo as principais: controle de zoonoses, higiene dos alimentos, trabalhos de laboratório, de biologia e as atividades experimentais (11).

Os princípios considerados base para a saúde pública veterinária estão relacionados com as ciências sociais e biológicas que se encontram na agricultura, saúde animal, educação, ambiente e na saúde humana (12).

\footnotetext{
4 A Organização Mundial de Saúde (OMS) (11) conceitua zoonose como "infecção ou doença infecciosa
} transmissível, em condições naturais, dos animais vertebrados ao homem". 
Dois grandes períodos citados por Schwabe (13) descrevem a atividade pública dentro da Medicina Veterinária. O primeiro período, que compreende o final do século XIX e início do século XX, possui como marco o contexto da higiene alimentar e foi com este fundamento que alguns veterinários alcançaram posições administrativas nos programas de saúde pública de inúmeros países. A segunda fase teve início após a Segunda Guerra Mundial e se definiu pela atividade centrada para a população, com o desenvolvimento de programas no controle de zoonoses através das agências de saúde pública. Em decorrência dessa nova interação com profissionais da medicina humana, os veterinários ocuparam espaços nas áreas técnicas e administrativas da saúde pública.

Nas escolas francesas de veterinária na primeira metade do século XIX, médicos e veterinários europeus desenvolveram, em parceria, pesquisas médicas comparadas nas áreas de anatomia e fisiologia. O crescente reconhecimento de tais estudos ocorreu em paralelo com a importância da Medicina Veterinária para a saúde humana. Tais estudos e o prosseguimento das pesquisas forneceram os princípios para a elaboração da revolução microbiológica. A Medicina Veterinária e a Medicina Humana foram fortemente ligadas através do acréscimo da pesquisa médica, influenciando 0 desenvolvimento voltado aos interesses da medicina humana mais do que aos da agricultura, em algumas escolas (13).

Considerada uma profissão cruzada, por voltar-se ao mesmo tempo para a saúde dos seres humanos e animais, a saúde pública veterinária apresenta uma natureza interdisciplinar (3).

O relatório da OMS, de 1975, descreve sobre a existência de uma grande quantidade de contribuições da Medicina Veterinária para a saúde humana, entre elas, a primeira fundamenta a mais básica função do veterinário sanitarista através da conexão com os animais e suas doenças, interligadas à saúde e bem-estar do homem. A incumbência relacionada diretamente com os animais inclui: vigilância em zoonoses; permuta de informações sobre pesquisas médicas veterinárias e a humanas, além da interligação e cooperação entre as organizações de saúde pública e veterinária; estudos epidemiológicos comparativos de enfermidades não infecciosas dos animais em 
referência aos humanos; substâncias tóxicas e venenos provenientes dos animais; vigilância sanitária e inspeção dos alimentos (14).

A Saúde Pública Veterinária deve estar combinada às áreas da saúde, agricultura e ambiente. Dessa forma, ela foi redefinida como "a soma de todas as contribuições para o bem-estar físico, mental e social dos seres humanos mediante a compreensão e a aplicação da ciência veterinária" (15).

Paim e Queiroz (10) dividem as atividades e responsabilidades do veterinário como membro da equipe de saúde pública nas esferas municipal, estadual e federal em três grupos: geral, especial e eventual. Como atividade geral, a cooperação desse profissional ocorre na participação do planejamento, coordenação, promoção e avaliação dos programas integrados de saúde. $\mathrm{Na}$ especial, ele atua na coordenação e o planejamento ocorre nos seguintes campos: controle de alimentos desde a sua produção até o comércio, no controle ou erradicação das zoonoses, programas de educação sanitária, avaliação epidemiológica e manutenção de dados estatísticos sobre as zoonoses. A atividade eventual é descrita por uma substituição temporária de um membro da equipe técnica que tenha se afastado de sua função, sendo assim necessário preencher o quadro de funcionários, o médico veterinário pode ocupar por tempo determinado tal função devido ao amplo conhecimento na área.

Neste contexto, o trabalho tem o objetivo de apresentar o papel e a importância do médico veterinário no Sistema Único de Saúde brasileiro, com base na legislação em vigor.

\section{Metodologia}

Tratou-se de um estudo de natureza qualitativa utilizando a análise bibliográfica e legislativa. A análise legislativa contemplou a Lei 8.080, de 19 de setembro de 1990, e para identificar o papel do médico veterinário na saúde pública, utilizou-se como método para a construção do artigo a pesquisa bibliográfica por meio do método descritivo.

Foram consultadas as bases de dados: Scientific Electronic Library Online (SCIELO), Scholar, Biblioteca Virtual em Saúde (BVS) e a Biblioteca Virtual em Medicina Veterinária e Zootecnia (BVS-VET). Também foram explorados periódicos do Conselho de Federal de Medicina Veterinária e Manuais do Ministério da Saúde e do Ministério da 
Agricultura e Pecuária do Brasil. Para o levantamento foram utilizadas as palavras chaves: "atuação do médico veterinário", "médico veterinário e saúde pública", isoladamente ou por associação entre elas, no sentido de selecionar os artigos científicos publicados.

Como critério de inclusão, privilegiou-se os artigos que explicitamente elencavam o médico veterinário na atividade de saúde pública e como critério de exclusão, os artigos que não mencionavam a atuação do médico veterinário na saúde pública.

A pesquisa bibliográfica foi realizada entre os dias 01 de setembro de 2015 e 01 de dezembro de 2015 e não determinou marco inicial temporal, englobando-se todo o universo de publicações, independentemente do ano.

\section{Resultados}

Foram encontrados nas bases de dados 21 trabalhos incluindo artigos de periódicos, teses de mestrado e doutorado. Atenderam ao critério de inclusão, 16 trabalhos que se relacionavam diretamente com o tema estudado.

Observou-se que a partir do ano de 2011, com a inserção do Médico Veterinário no Núcleo de Apoio à Saúde da Família (NASF), houve um incremento de publicações na área de Saúde Pública Veterinária (2), (16), (17) e (18), embora o número total de trabalhos seja baixo.

No que diz respeito à educação veterinária e as diretrizes curriculares das universidades que oferecem o curso de Medicina Veterinária, vários autores consideram como um dos pontos fundamentais para a formação de novos veterinários sanitaristas para o mercado de trabalho (13), (19), (20), (21) e (22).

A Lei 8.080/90, nos artigos $6^{\circ}$ e 12 ao 18, relaciona as competências e atribuições comuns nas três esferas de poder nas vigilâncias sanitária, epidemiológica e ambiental e a formação das comissões intersetoriais e permanentes (6). É justamente no que se refere a essas vigilâncias e na participação da formação das políticas e estratégias da saúde pública nacional que se insere o médico veterinário (20). 


\section{Discussão}

Apesar de a Medicina Veterinária estar diretamente envolvida com as necessidades básicas de saúde do ser humano, os seus objetivos são difusos havendo uma incompatibilidade do papel social indexado a profissão e a educação veterinária que não consegue acompanhar essa tendência (13).

Do ponto de vista educacional, as diretrizes curriculares enfatizam a necessidade de envolver esse profissional a desenvolver competências para integrar as equipes multiprofissionais no contexto do SUS (19).

A pesquisa documental realizada por Bürger (20), em 2010, no estado de São Paulo, tomando como fonte as matrizes curriculares utilizadas nos cursos de graduação em Medicina Veterinária, constatou que as diretrizes curriculares não contemplam de forma compatível à área de atuação da Saúde Pública Veterinária, direcionando os estudantes para uma formação individual e curativa, sem consciência do significado e a importância da atuação do profissional na área de Saúde Pública Veterinária.

$\mathrm{Na}$ educação dos profissionais de saúde, entre eles o médico veterinário sanitarista, observa-se que a formação generalista é pouco valorizada, sendo substituída pelo subjetivismo da relação profissional da saúde-paciente pela objetividade tecnológica das ciências da saúde. A hierarquização e a desintegração do conhecimento dão origem a segregação da teoria e prática, fragmentam o conhecimento e impedem que este profissional busque espaços que vão além dos aprendizados das universidades, não enxergando novos campos de atuação que condizem com as realidades das populações (21).

O baixo número de publicações sobre o tema da saúde sública veterinária é justificado, pela forma de ensino das universidades no curso de Medicina Veterinária, com cargas horárias reduzidas de matérias sobre a medicina veterinária preventiva e saúde pública, fornecendo apenas noções genéricas e superficiais aos estudantes. Tais fatores impossibilitam o reconhecimento dos mesmos a cerca da influência dos elementos sociais sobre a saúde pública (22).

O campo da Medicina Veterinária é vasto e as oportunidades para a saúde pública veterinária são ilimitadas, porém o seu maior desafio é ser capaz de aplicar a grande quantidade de resultados de pesquisas disponíveis e o conhecimento técnico dotado 
para melhorar a desigualdade social, contribuindo para o desenvolvimento nacional (2). Para enfrentar esses desafios é importante a cooperação, por meio de parcerias internacionais, para informar e preparar os profissionais sobre as doenças pandêmicas, que atingem mais de um país ou de um continente, e sobre a necessidade de uma nova revisão dos currículos de graduação com o objetivo de capacitar os alunos, futuros veterinários, para os desafios da saúde global (23).

No Brasil, devido à ausência de articulação social e política, baixa expressividade e participação do veterinário sanitarista nos Conselhos Municipais de Saúde, há uma queda da representatividade da Medicina Veterinária no serviço de saúde, circunstância que deve ser atenuada o quanto antes, para possibilitar uma maior cooperação desses profissionais na formação das políticas e estratégias da saúde pública nacional (23).

$\mathrm{Na}$ Lei 8.080/1990, artigos 12 a 14, o veterinário sanitarista pode participar das Comissões Intersetoriais de âmbito nacional, a fim de, articular políticas e programas de interesse para a saúde pública, em especial, nas seguintes atividades: saneamento e meio ambiente; vigilância sanitária; e ciência e tecnologia. Outra participação importante desse profissional é na Comissão Permanente de integração entre os serviços de saúde e as instituições de ensino profissional e superior (6).

As zoonoses são um dos principais agravantes à saúde pública mundial, e por se tratarem de doenças transmitidas por animais silvestres e domésticos aos humanos, necessitam de estratégias de prevenção e controle inovadoras que requerem o esforço combinado de vários campos da saúde. Por exemplo, os veterinários, médicos e profissionais da saúde pública atuando em conjunto nas três principais áreas da saúde: a saúde individual, saúde coletiva e na pesquisa médica comparativa. Na saúde individual o veterinário conseguiria estabelecer e assessorar os médicos sobre os potenciais de risco da transmissão de doenças zoonóticas, especialmente em casos que envolvam pacientes de alto risco, como os imunocomprometidos. Na saúde coletiva, ameaças de doenças zoonóticas deveriam ser adereçadas por meio de sistemas de vigilância que incluam animais domésticos e silvestres e populações humanas, a fim de guiar medidas mais eficientes de controle. No campo da pesquisa, a colaboração entre médicos e veterinários na medicina comparativa poderia melhorar o entendimento das interações agente-hospedeiro (24). 
Com o aumento da incidência das doenças infecciosas, parasitárias e considerando que cerca de $80 \%$ das doenças infecciosas emergentes e reemergentes 5 são de origem animal, em 2009, durante o III Congresso Nacional de Saúde Pública Veterinária e o I Encontro Internacional de Saúde Pública Veterinária, a representante da Organização Pan-Americana da Saúde/Organização Mundial da Saúde (OPAS/OMS) Christina Schneider, comunicou que na América Latina um bilhão de pessoas convivem com 150 milhões de cães, 500 milhões de bovinos e 150 espécies de morcegos, ressaltando a tarefa do médico veterinário na interface das relações dessas espécies (23). Essa relação direta com os animais fez com que o médico veterinário se tornasse uma peça fundamental no controle de doenças e na manipulação de fatores ambientais que se articulam com a vida das pessoas, tornando-se essencial a aproximação desse profissional com os indivíduos e seus domicílios, nos quais se inserem a vida das famílias, seus animais e outros atores e composições ambientais (25).

A multicausalidade das doenças levou a crer que a saúde necessita do conhecimento de muitas profissões, atividade conhecida como integralidade, e materializar este entendimento passou a ser tarefa dos profissionais atuantes no segmento da saúde pública (25).

$\mathrm{Na}$ Lei 5.517, de 1968, que dispõe sobre o exercício do médico veterinário, nos artigos $5^{\circ}$ e $6^{\circ}$ verifica-se que é competência do veterinário sanitarista o estudo e a aplicação de medidas de saúde pública no tocante às doenças de animais transmissíveis ao homem e a inspeção e a fiscalização sob o ponto-de-vista sanitário, higiênico e tecnológico de produtos de origem animal (26).

O Conselho Federal de Medicina Veterinária (CFMV) em união com o Conselho Nacional de Saúde (CNS) do Ministério da Saúde (MS) modificou a percepção e reconhecimento da Medicina Veterinária como profissão vinculada à saúde, por meio da Resolução no 38/1992 do Conselho Nacional de Saúde-CNS/MS, alterada pela Resolução nº 218/1997-CNS/MS e republicada como Resolução no 287/1998-CNS/MS com a inserção do veterinário na atuação do Conselho de Saúde. Desde então, o

\footnotetext{
5 O conceito de doenças infecciosas emergentes e reemergentes surgiu a partir dos anos 1990 e representa o crescente processo de disseminação dos microrganismos e sua interação com o homem, devido a mudança expressiva do estilo de vida e do desenvolvimento econômico, principalmente com as políticas de industrialização (27). O conceito dessas doenças foi definido por Satcher (28) como "aquelas só recentemente identificadas na população humana ou já existentes, mas que rapidamente aumentaram sua incidência e ampliaram sua distribuição geográfica".
} 
Médico Veterinário tem demonstrado sua capacidade e competência para atuar nas equipes de Vigilâncias Epidemiológica, Sanitária e Ambiental (29).

Tornou-se evidente a necessidade da organização de um modelo de atenção com base na qualidade e estilo de vida das pessoas e do seu meio ambiente, tal como na relação da equipe de saúde com as comunidades e com os seus núcleos sociais primários. A reorganização da atenção primária, particularmente por meio do Programa de Saúde da Família (PSF), vinculada a vigilância sanitária pode ter sido a intervenção mais ampla realizada no Brasil visando à modificação do modelo de atenção hegemônico (30).

Com o intuito de fortalecer as ações de caráter preventivo na saúde, durante os anos 1990, o Ministério da Saúde investiu em programas de ações básicas, estimulando a formulação do PSF como parte da estratégia de reorganização do próprio modelo de atenção, com o foco na promoção da saúde (16).

O Ministério da Saúde criou os Núcleos de Apoio à Saúde da Família (NASF), mediante a Portaria n 154/GM, de 24 de janeiro de 2008 (31), e logo em seguida, a Portaria $\mathrm{n}^{\circ} 2488$ de 21 de outubro de 2011 acrescentou o médico veterinário na composição das equipes dos NASFs, permitindo que os gestores municipais de saúde incorporassem o esse profissional no quadro de atuação para a saúde da família (32).

A inserção do veterinário foi um importante marco para esta classe profissional e para a sociedade. Porém, para que haja a real participação nas equipes de atenção básica e a efetivação da proposta de atender às necessidades sócio-epidemiológicas das populações cobertas pelo sistema, é indispensável o comprometimento de todas as esferas do poder público de uma maneira cada vez mais municipalizada e politicamente independente (17).

O Conselho Regional de Mato Grosso por meio da Resolução CRMV-MT nำ21, de 14 de janeiro de 2013, estabeleceu as competências do Médico Veterinário quanto às condutas e procedimentos específicos de cada intervenção do profissional Médico Veterinário no Núcleo de Apoio a Saúde da Família (NASF) em sua região:

- I - Fazer visitas domiciliares educativas e orientativas, não considerando consulta clínica, para o diagnóstico de risco que envolva o homem, animais e o ambiente; 
- Il - Fazer prevenção, controle e diagnóstico situacional de risco de doenças transmissíveis por alimentos e animais vertebrados e/ou invertebrados de casos específicos;

- III - Desenvolver ações educativas com foco na promoção, prevenção e controle de doenças de caráter zoonóticas e demais riscos por determinados animais sinantrópicos, venenosos, peçonhentos ou não;

- IV - Fazer estudos e pesquisas em saúde pública de modo contínuo e sistemático, que favoreçam a qualidade da atenção em saúde, identificando emergências epidemiológicas de potencial zoonótico ou que envolvam animais invertebrados como fonte de infecção;

- V - Identificar e orientar sobre os riscos de contaminação por substâncias tóxicas (produtos de uso veterinário);

- VI - Elaborar projetos de prevenção de doenças e promoção à saúde, por meio de discussões periódicas junto às Equipes de Saúde da Família (33).

Essas orientações se aplicam apenas na região de Mato Grosso, porém servem de base para as outras regiões do Brasil.

O SUS possui como princípio um sistema de organização baseado na universalidade de acesso aos serviços de saúde, integralidade e igualdade de assistência à saúde e participação da comunidade (6).

Ao analisar o papel do Médico Veterinário no SUS, em especifico na Lei 8.080/1990, verifica-se a execução de ações desse profissional na Vigilância em Saúde ${ }^{6}$. A criação da Secretária de Vigilância em Saúde do Ministério da Saúde (SVS/MS) ocorreu em junho de 2003 pelo decreto n 4.726, em substituição ao Centro Nacional de Epidemiologia, da Fundação Nacional de Saúde (CENEPI) e passou a coordenar o Sistema Nacional de Vigilância em Saúde. A partir da sua criação, todas as ações de vigilância, prevenção e controle de doenças e promoção em saúde se reuniram em uma única estrutura do Ministério da Saúde, responsável pela coordenação nacional das ações, executadas pelo SUS e compartilhadas segundo as atribuições de

\footnotetext{
6 Vigilância em saúde é composta pela vigilância das doenças transmissíveis, das doenças não transmissíveis e da análise da situação de saúde. Compreende as ações de Vigilância Epidemiológica, Sanitária, Ambiental e Saúde do Trabalhador, com o objetivo de promover o conhecimento, detectar e prevenir qualquer mudança nos fatores determinantes e condicionantes de saúde individual e coletiva (35).
} 
cada esfera de governo, com gestores estaduais e municipais. A fundação da SVS é de grande importância para a saúde pública veterinária, pois é onde são realizadas as medidas de prevenção, promoção da saúde, notificação e controle de zoonoses (34).

As atribuições da vigilância sanitária ${ }^{7}$, epidemiológica ${ }^{8}$ e ambiental estão descritas dentre as competências das três esferas de poder no SUS, presentes nos artigos 16, 17 e 18 da Lei 8.080/1990. A união é responsável por: participar na formulação e na implementação das políticas das três vigilâncias; coordenar e participar na execução das ações de vigilância epidemiológica; estabelecer normas e executar a vigilância sanitária de portos, aeroportos e fronteiras, podendo a execução ser complementada pelos Estados, Distrito Federal e Municípios; além de estabelecer critérios, parâmetros e métodos para o controle da qualidade sanitária de produtos, substâncias e serviços de consumo e uso humano (6).

Ao Estado compete coordenar, executar ações e implementar serviços das vigilâncias, em caráter complementar, e colaborar com a União na execução da vigilância sanitária de portos, aeroportos e fronteiras. Os municípios executam os serviços das vigilâncias e colaboram com a União e os Estados na execução da vigilância sanitária de portos, aeroportos e fronteiras (6).

Os órgãos participantes desse Sistema são: Ministério da Saúde, a Agência Nacional de Vigilância Sanitária (ANVISA), o Conselho Nacional de Secretários Estaduais de Saúde (CONASS), o Conselho Nacional de Secretários Municipais de Saúde (CONASEMS), os Centros de Vigilância Sanitária Estaduais, do Distrito Federal e Municipal (VISAs), os Laboratórios Centrais de Saúde Pública (LACENS), o Instituto Nacional de Controle de Qualidade em Saúde (INCQS), a Fundação Oswaldo Cruz (FIOCRUZ) e os Conselhos Estaduais, Distritais e Municipais de Saúde, em relação às ações de vigilância sanitária (36).

$\mathrm{Na}$ vigilância ambiental o médico veterinário está apto para opinar sobre os seguintes assuntos: a contaminação dos recursos hídricos; a disposição de dejetos e resíduos sólidos; a contaminação química através do uso de agrotóxicos, inseticidas,

\footnotetext{
${ }^{7}$ A vigilância sanitária pode ser descrita como "um conjunto de ações capaz de eliminar, diminuir ou prevenir riscos à saúde e de intervir nos problemas sanitários decorrentes do meio ambiente, da produção e circulação de bens e da prestação de serviços de interesse da saúde" (6).

${ }^{8}$ A vigilância epidemiológica é formada por "um conjunto de ações que proporcionam o conhecimento, a detecção ou prevenção de qualquer mudança nos fatores determinantes e condicionantes de saúde individual ou coletiva, com a finalidade de recomendar e adotar as medidas de prevenção e controle das doenças ou agravos" (6).
} 
parasiticidas; a destruição da flora e da fauna; a perda da biodiversidade; os medicamentos, promotores de crescimento e hormônios utilizados na alimentação animal; e os modelos de exploração pecuária (18).

\section{Considerações finais}

O médico veterinário possui um conhecimento interdisciplinar e é importante no contexto da saúde pública por atuar na prevenção e promoção da saúde humana, por meio da notificação, avaliação epidemiológica e controle das zoonoses, da inspeção e fiscalização sanitária, higiênica e tecnológica de produtos de origem animal e programas de educação sanitária e ambiental.

Considerando que a saúde do homem constitui uma das principais preocupações do médico veterinário no exercício de sua função, buscando sempre a melhoria da qualidade de vida da sociedade humana. Porém, este profissional ainda não é reconhecido pela sociedade como um agente público de saúde o que dificulta a sua inserção nos principais programas de saúde pública, como exemplo o NASF, e na conquista de novos espaços nas áreas da saúde pública. No SUS, o médico veterinário pode atuar nas vigilâncias em saúde, nas Comissões Intersetoriais e Permanente e nas Direções Nacional, Estadual e Municipal, presentes nos artigos $6^{\circ}$ e 12 ao 18 da Lei Orgânica da Saúde.

Por fim, a formação do veterinário nas instituições de ensino no Brasil não é dada a devida importância às disciplinas de saúde pública, seja pela forma como é administrada nas salas de aula ou por cargas horárias reduzidas. A formação dos estudantes é direcionada, quase que exclusivamente, para a área clínica o que dificulta 0 autorreconhecimento por parte desse profissional no campo da saúde e na conscientização da importância do exercício profissional na vasta e dinâmica área da Saúde Pública Veterinária. 


\section{Referências}

1. Germiniani CLB. A história da medicina veterinária no Brasil. Archives of Veterinary Science 3 (1): 1-8, 1998 [Acesso em 15 out 2015], Disponível em: http://ojs.c3sl.ufpr.br/ojs/index.php/veterinary/article/viewArticle/3732.

2. Da costa HX, Linhares GFC. A importância do médico veterinário no contexto de saúde pública [Dissertação]. Goiânia: Programa de Pós-Graduação em Ciência Animal da Escola de Veterinária da Universidade Federal de Goiás; 2011. [Acesso em 17 set 2015]. Disponível em: http://portais.ufg.br/up/67/o/Seminario2011 Herika Costa 1.pdf.

3. Pfuetzenreiter MR, Zylbersztajn A, Avila-pires FD. Evolução histórica da medicina veterinária preventiva e saúde pública. Ciência Rural 34 (5): 1661-1668, 2004 [Acesso em 17 out 2015]. Disponível em: http://www.scielo.br/pdf/cr/v34n5/a55v34n5.pdf.

4. Cruz HM, Acha PN. La función del médico veterinario en la salud de la comunidad en la América Latina. Educación Médica y Salud 6 (3-4): 256-28,1972 [Acesso em 15 out 2015]. Disponível em: http://hist.library.paho.org/Spanish/EMS/39395.pdf.

5. Teixeira CF, Paim JS, Vilasbôas ALQ. SUS, modelos assistenciais e vigilância da saúde. In: Rozenfeld S, organizadora. Fundamentos da Vigilância Sanitária. Rio de Janeiro: FIOCRUZ; 2000. p.49-60.

6. Brasil. Lei no 8.080 , de 19 de setembro de 1990. Dispõe sobre as condições para a promoção, proteção e recuperação da saúde, a organização e o funcionamento dos serviços correspondentes e dá outras providências. Diário Oficial da República Federativa do Brasil. Brasília, 20 set 1990 [Acesso em 17 set 2015]. Disponível em: http://www.planalto.gov.br/ccivil 03/Leis/L8080.htm.

7. Paim, JS. A Reforma Sanitária e o CEBES / Jairnilson Silva Paim. Rio de Janeiro: CEBES; 2012. [Acesso em 17 set 2015]. Disponível em: http://cebes.org.br/site/wpcontent/uploads/2014/11/E-Book-1-A-Reforma-Sanit\%C3\%A1ria-Brasileira-e-oCEBES.pdf.

8. Brasil. Constituição da República Federativa do Brasil, 8 de outubro de 1988. Brasília: Senado Federal, 2008 [Acesso em 17 set 2015]. Disponível em: http://www.planalto.gov.br/ccivil 03/constituicao/ConstituicaoCompilado.htm.

9. Cunha JRA. Avanços e retrocessos do direito à saúde no Brasil: uma esperança equilibrista [Dissertação]. Brasília: Universidade de Brasília; 2014. [Acesso em 17 out 2015]. Disponível em: http://repositorio.unb.br/handle/10482/16525.

10. Paim GV, Queiroz JC. Saúde pública veterinária. Arquivos da Faculdade de Higiene e Saúde Pública da Universidade de São Paulo 18 (1-2): 111-118, 1964 [Acesso em 22 set 2015]. Disponível em: http://www.revistas.usp.br/afhsp/article/view/85767. 
11. World Health Organization. Joint WHO/FAO Expert Group on Zoonoses: Report on the First Session. Geneva: 1951. [Acesso em 24 set 2015]. Disponível em: http://apps.who.int/iris/handle/10665/40155.

12. Arámbulo $P, R u i ́ z A$. Situación actual y futura de la medicina veterinaria. Educación Médica y Salud 26 (2): 263-276,1992 [Acesso em 24 set 2015]. Disponível em: http://hist.library.paho.org/Spanish/EMS/11641.pdf.

13. Schwabe CW. Veterinary medicine and human health. 3 ed. Baltimore: Williams e Wilkins; 1984.

14. World Health Organization. The veterinary contribution to public health practice: Report of a Joint FAO/ WHO Expert Committee on Veterinary Public Health. Geneva: 1975. [Acesso em 24 set 2015]. Disponível em: http://apps.who.int/iris/handle/10665/38156.

15. World Health Organization. Future Trends in Veterinary Public Health: Report of a WHO Study Group. Geneva: 2002. [Acesso em 24 set 2015]. Disponível em: http://www.who.int/zoonoses/resources/zoonoses vph/en/.

16. Costa MML. O Núcleo de Apoio à Saúde da Família (NASF): contextualização de sua implantação na atenção básica à saúde no Brasil [Dissertação]. Brasília: Instituto de Ciências Humanas, Universidade de Brasília; 2013. [Acesso em 14 set 2015]. Disponível em: http://bdm.unb.br/handle/10483/4945.

17. Junior AMF, Fonseca AG. A inclusão do Médico Veterinário na Atenção Básica à Saúde da Família. In: VII CONNEPI-Congresso Norte Nordeste de Pesquisa e Inovação, 2012. Palmas. [Acesso em 28 set 2015]. Disponível em: http://propi.ifto.edu.br/ocs/index.php/connepi/vii/paper/view/5050.

18. Possamai MHP. O papel do médico veterinário na educação e formação na vigilância ambiental em saúde. Revista Electrónica de Investigación y Docencia (REID). 2011 [Acesso em 08 out 2015], Número Monográfico: 59-73. Disponível em: http://revistaselectronicas.ujaen.es/index.php/reid/article/view/1139.

19. Brasil. Conselho Nacional de Educação. Resolução CNE/CES № 1, de 18 de fevereiro de 2003. Institui Diretrizes Curriculares Nacionais dos Cursos de Graduação em Medicina Veterinária. Diário Oficial da União. Brasília, 20 fev 2003. [Acesso em 18 out 2015]. Disponível em: http://portal.mec.gov.br/cne/arquivos/pdf/ces012003.pdf.

20. Bürger KP. O ensino de saúde pública veterinária nos cursos de graduação em medicina veterinária do Estado de São Paulo [Tese]. Jaboticabal: Faculdade de Ciências Agrárias e Veterinárias, Universidade Estadual Paulista; 2010. [Acesso em 15 out 2015]. Disponível em: http://repositorio.unesp.br/handle/11449/103824.

21. Ferreira RC, Fiorini VML, Crivelaro E. Formação profissional no SUS: o papel da Atenção Básica em Saúde na perspectiva docente. Revista Brasileira de Educação 
Médica 34 (2): 207-15, 2010. [Acesso em 05 out 2015],. Disponível em: http://www.scielo.br/pdf/rbem/v34n2/a04v34n2.pdf.

22. Pfuetzenreiter MR. O ensino da medicina veterinária preventiva e saúde pública nos cursos de medicina veterinária: estudo de caso realizado na Universidade do Estado de Santa Catariana [Tese]. Florianopólis: Programa de Pós-Graduação em Educação, Universidade Federal de Santa Catarina; 2003. [Acesso em 15 out 2015]. Disponível em: https://repositorio.ufsc.br/handle/123456789/85822.

23. Napoli L. O Contexto do Médico Veterinário na Saúde Pública Contemporânea. Conselho Regional de Medicina Veterinária do Paraná. 2011: 1-5. [Acesso em 17 out 2015]. Disponível em: http://www.crmv-pr.org.br/?p=imprensa/artigo detalhes\&id=81\#.

24. Kahn LH. Confronting zoonoses, linking human and veterinary medicine. Emerging Infectious Diseases. 2006 [Acesso em 19 out 2015], 12 (4): 556-561. Disponível em: http://wwwnc.cdc.gov/eid/article/12/4/pdfs/05-0956.pdf.

25. Comissão Nacional de Saúde Pública Veterinário do Conselho Federal de Medicina Veterinária. O Médico Veterinário, a Estratégia de Saúde da Família e o NASF. Revista CFMV. 48 (1): 9-14, 2009

26. Brasil. Presidência da República. Lei oㅜ 5.517, de 23 de outubro de 1968. Dispõe sobre o exercício da profissão de médico-veterinário e cria os Conselhos Federal e Regionais de Medicina Veterinária. Brasília, 23 out 1968 [Acesso em 06 out 2015]. Disponível em: http://presrepublica.jusbrasil.com.br/legislacao/110145/lei-5517-68.

27. Centers for Disease Control and Prevention. Addressing emerging infectious disease threats: a prevention strategy for the United States (Executive Summary). Georgia:

MMWR, 43: 1-16,1994. [Acesso em 17 out 2015]. Disponível em: http://www.cdc.gov/mmwr/PDF/rr/rr4305.pdf.

28. Satcher D. Emerging Infectious: Getting Ahead of the Curve. Emerging Infectious Diseases 1 (1): 1-6, 1995 [Acesso em 19 out 2015], Disponível em: http://wwwnc.cdc.gov/eid/article/1/1/pdfs/95-0101.pdf.

29. Souza PCA. A inserção do médico veterinário na área de saúde. Revista do CFMV. 2004; 16: 5-7.

30. Paim JS. Atenção à saúde no Brasil. Saúde no Brasil - Contribuições para a Agenda de Prioridades de Pesquisa 1 (B): 15-44, 2004 [Acesso em 06 out 2015].. Disponível em: http://bvsms.saude.gov.br/bvs/publicacoes/Saude.pdf.

31. Brasil. Ministério da Saúde. Portaria $n^{\circ}$ 154, de 24 de janeiro de 2008. Cria os Núcleos de Apoio à Saúde da Família - NASF. Diário Oficial da União. Brasília, 04 mar 2008. [Acesso em 02 nov 2015]. Disponível em:

http://bvsms.saude.gov.br/bvs/saudelegis/gm/2008/prt0154 2401 2008.html. 
32. Brasil. Ministério da Saúde. Portaria $n^{\circ} 2.488$, de 21 de outubro de 2011. Aprova a Política Nacional de Atenção Básica, estabelecendo a revisão de diretrizes e normas para a organização da Atenção Básica, para a Estratégia Saúde da Família (ESF) e o Programa de Agentes Comunitários de Saúde (PACS). Diário Oficial da União [Internet] Brasília, 24 out 2011. [Acesso em 02 nov 2015]. Disponível em: http://bvsms.saude.gov.br/bvs/saudelegis/gm/2011/prt2488 2110 2011.html.

33. Brasil. Conselho Regional de Medicina Veterinária do Estado de Mato Grosso. Resolução CRMV-MT oㅡ 21, de 14 de janeiro de 2013 (Atualizada). Estabelece as competências do Médico Veterinário quanto às condutas e procedimentos específicos de cada intervenção do profissional Médico Veterinário no Núcleo de Apoio a Saúde da Família- NASF no estado de Mato Grosso [Internet]. [Acesso em 02 nov 2015]. Disponível em: http://www.crmv-mt.org.br/images/bt-arise/Resolucoes/021-2013Atualizada.pdf.

34. Secretária de Vigilância em Saúde. Vigilância em saúde no SUS: fortalecendo a capacidade de resposta aos velhos e novos desafios. Brasília: Ministério da Saúde; 2006. [Acesso em 02 nov 2015]. Disponível em: http://livros01.livrosgratis.com.br/ms000273.pdf.

35. Noronha JC, Penna GO. Saúde da Família e Vigilância em Saúde: em busca da integração das práticas. Revista Brasileira Saúde da Família 16: 4-9, 2007 [Acesso em 02 nov 2015], Disponível em:

http://189.28.128.100/dab/docs/publicacoes/revistas/revista saude familia16.pdf.

36. Guimarães FF, Baptista AAS, Gustavo PMGM, Langoni H. Ações da vigilância epidemiológica e sanitária nos programas de controle de zoonoses. Revista Veterinária e Zootecnia 17 (2): 151-162, 2010 [Acesso em 05 nov 2015].. Disponível em: http://www.fmvz.unesp.br/rvz/index.php/rvz/article/view/9. 